\title{
NUCLEAR FRAGMENTATION AND THE NUMBER OF PARTICLE TRACKS IN TISSUE
}

\author{
A. L. Ponomarev ${ }^{1,2, *}$ and F. A. Cucinotta ${ }^{1}$ \\ ${ }^{1}$ NASA Lyndon B. Johnson Space Center, Houston TX 77058, USA \\ ${ }^{2}$ U.S.R.A., Division and Life Space Sciences, Houston TX 77058, USA
}

For high energy nuclei, the number of particle tracks per cell is modified by local nuclear reactions that occur, with large fluctuations expected for heavy ion tracks. Cells near the interaction site of a reaction will experience a much higher number of tracks than estimated by the average fluence. Two types of reaction products are possible and occur in coincidence; projectile fragments, which generally have smaller charge and similar velocity to that of the projectile, and target fragments, which are produced from the fragmentation of the nuclei of water atoms or other cellular constituents with low velocity. In order to understand the role of fragmentation in biological damage a new model of human tissue irradiated by heavy ions was developed. A box of the tissue is modelled with periodic boundary conditions imposed, which extrapolates the technique to macroscopic volumes of tissue. The cross sections for projectile and target fragmentation products are taken from the quantum multiple scattering fragmentation code previously developed at NASA Johnson Space Center. Statistics of fragmentation pathways occurring in a cell monolayer, as well as in a small volume of $10 \times 10 \times 10$ cells are given. A discussion on approaches to extend the model to describe spatial distributions of inactivated or other cell damage types, as well as highly organised tissues of multiple cell types, is presented.

\section{INTRODUCTION}

Heavy ions originating from GCR (galactic cosmic rays) are a major concern for astronaut health during deep space mission to the Moon and Mars. The spectrum of heavy ions is well known in the regions of space of exploration concern: near the Earth, the deep space between Mars and Earth, and the Moon and Mars surface ${ }^{(1)}$. On a $1000 \mathrm{~d}$ Mars mission the total radiation dose received by an astronaut will amount to $\approx 1 \mathrm{~Sv}$. Heavy ions are a significant part of this dose. During a 3 y mission to Mars, on average every cell in human body will receive one heavy ion ${ }^{(2)}$. This is a very ballpark figure, as it does not take into account cell proliferation, and cell repair, which differs significantly among human body organs. The heavy ion component of GCR undergo nuclear reactions in design materials, or human tissue $e^{(1,3-6)}$. The attenuation of the flux of heavy ions by available shielding materials is poor because of secondary radiation, and not cost- and mass-efficient. The depth-dependence of this attenuation for various materials is well-studied and the data are available ${ }^{(5,6)}$. The heavier components can inflict more biological damage as characterised by their biological efficiency. There is always an uncertainty in the estimate of biological damage due to the complexity of biological systems. The primary motivation of this work is to prepare the ground to more precise biological prediction of the potential radiation damage from space heavy ions.

The mathematical modelling of the effects of radiation, especially heavy ions, is conducted now

*Corresponding author: aponomar@ems.jsc.nasa.gov in a wide range of molecular, subcellular, and cellular structures. Here the emphasis will be on the scale leading to the analysis of tissue effects from these ions. This is an introductory work, in which the physical process of ion passage through material (tissue) will be addressed, as well as a preliminary model of tissue will be proposed. The applications of this model to the studies of cell apoptosis and necrosis will be discussed in the Conclusions.

The novelty of this approach is the consideration of nuclear reaction in tissue, which may increase the flux of particles within the cells where these reactions occur. Some fraction of cells should be affected by fragments only, which are mostly lighter particles (e.g. protons and neutrons) and can cause mutagenesis and carcinogenesis. The tissue radiation properties are similar to water, for which the fragmentation cross sections are known. The tissue box model employs the quantum multiple scattering fragmentation (QMSFRG) model ${ }^{(7-9)}$. The biological structure does not affect the probability of the nuclear reactions, only its density. The fragmentation products of heavy ions can be numerous due to the build-up of protons and neutrons along with other target and projectile fragments. Target and projectile fragments have a sufficiently long range in tissue, which results in the delivery of their total energy over several cell diameters for target fragments and hundreds or thousands for the more energetic projectile fragments.

The biological damage from high-LET radiation is known to be qualitatively and quantitatively different from that of low-LET including the role of clustered DNA damage. The NASARadiationTrackImage $^{(}$model was previously proposed ${ }^{(3,4)}$ to 
examine the mechanistic consequences of high-LET radiation. The main conclusion was that the DSB RBE is the same for high-LET as for low-LET, but the clustering of damage that occurs during highLET leads to the preponderance of shorter DNA fragments produced by such radiation. The clustering of DNA damage can lead to complex chromosomal aberrations and small DNA deletions not visible in chromosomal aberration experiments.

Herein the goal will be to score the number of hits per cell both from the impinging particles and their fragments to prepare the ground for the studies of tissue damage including cell apoptosis and necrosis. The statistics of hits may depend not only on the incident radiation and nuclear reaction pathways, but also on cell arrangements in tissue. This opens a venue for new questions that this model will attempt to answer: (1) what is the real flux of particles within the human tissue, and how this flux is affected by nuclear reactions, and by a different pattern of cell organisation in tissue; (2) what is the statistics of particle hits in a particular type of tissue.

This work gives a preliminary answer to these questions based on a simple cell matrix model. Precise cellular maps are needed to refine the presented data in the future. To produce a complete cellular matrix of an individual human organ is currently unrealistic. A recent attempt to produce a cellular map of Drosophila fly brain map was done in Ref. (10), where it was shown that even for such a small biological object, mapping the entire organ is difficult. Therefore, the focus is on a small sample of tissue, and then try to scale-up the predicted results obtained on a cell matrix with $5 \times 5 \times 5$ to $10 \times 10 \times 10$ cells. A similar approach is employed in polymer science where polymer melts are studied on the microscopic level first, and then in a small cube of polymer melt. Such an approach accepted to model the complexity of synthetic polymers. The extrapolation is then made to the bulk. The complexity of biological systems is even more staggering. This preliminary model will open a door to the new areas of biological systems modelling.

\section{METHODS}

\section{Tissue box}

First, a slab of physical space is introduced, where a model of tissue as well as physical processes will take place. This slab a tissue is called the tissue representative box (TRB). This cube of physical space of a given size will have a sample of the simulated tissue, incident particles and various processes related to the action of radiation on tissue. Particularly, the focus is on ion fragmentation, and cell killing. Next, a cubic lattice is introduced, or grid that has $8 \mu \mathrm{m}$ per lattice node. The number of lattice sites in
TRB, as well as the size of the node can be controlled through the interface (Figure 1). A typical dimension for the whole cube is $80 \mu \mathrm{m}$, which will correspond up to $\sim 10$ cells rows along any axis.

The intent of the model is to include a collection of different tissue maps with individual distributions for cell sizes and shapes, as well as their spatial density. This preliminary version of the algorithm has two options: a monolayer corresponding to epithelium, and a slab of tissue representing an organ with a relatively homogenous cell matrix. Cell sizes can vary independently of the grid size.

\section{Periodic boundary conditions}

As a way to extrapolate this model to larger pieces of tissue the periodic boundary conditions (PBCs) were imposed on the TRB. PBC produces an infinite pattern of cells translated indefinitely in $x$, and $y$ directions by the multiples of the box size $L$, which is given by

$$
L=N^{*} d \sim 10^{*} 8=80 \mu \mathrm{m},
$$

where $N$ is the number of grid nodes (Figure 1) along one axis, and $d$ is the physical dimension of a grid node. For a multi-layer of cells $N=5$ and the TRB period of $L=40 \mu \mathrm{m}$ were used.

A number of impinging particles is generated in TRB proportional to the flux. The entry coordinates of particles $(x, y)$ 's locations (located at the entry pane of the TRB) are translated indefinitely along the $\pm(x, y)$ axes, in the same manner as cells. This reproduces a macroscopic slab of tissue.

The PBC were not imposed in the $z$ direction because particles may enter and exit the cube in the $z$ direction. Even though the length of the cube in that direction is $L$, the depth in tissue is arbitrary, as particles like $\mathrm{Fe}$ ions practically do not lose their energy while traversing the tissue. The products of fragmentation have the same probability to occur at any depth in tissue.

When fragmentation occurs some products can exit through the panels of the original TRB. When a particle exits the cube, exactly the same particle re-enters the cube at the opposite face, as if the whole space shifted by the period $L$ in the direction opposite to the particle motion.

The reason why $\mathrm{PBCs}$ are important in simulations of particles traversing cells is that it is a feasible method to extrapolate data from the microscopic box for the bulk of tissue. The application of PBCs is equivalent to tiling the entire physical space with identical boxes. In real tissue the stochastic events are different in these boxes. However, since the stochastic events are similar in the original box to the events anywhere in tissue in terms of their probabilities, distribution functions, distribution moments or other parameters, the result of the simulation of 


\section{A. L. PONOMAREV and F. A. CUCINOTTA}

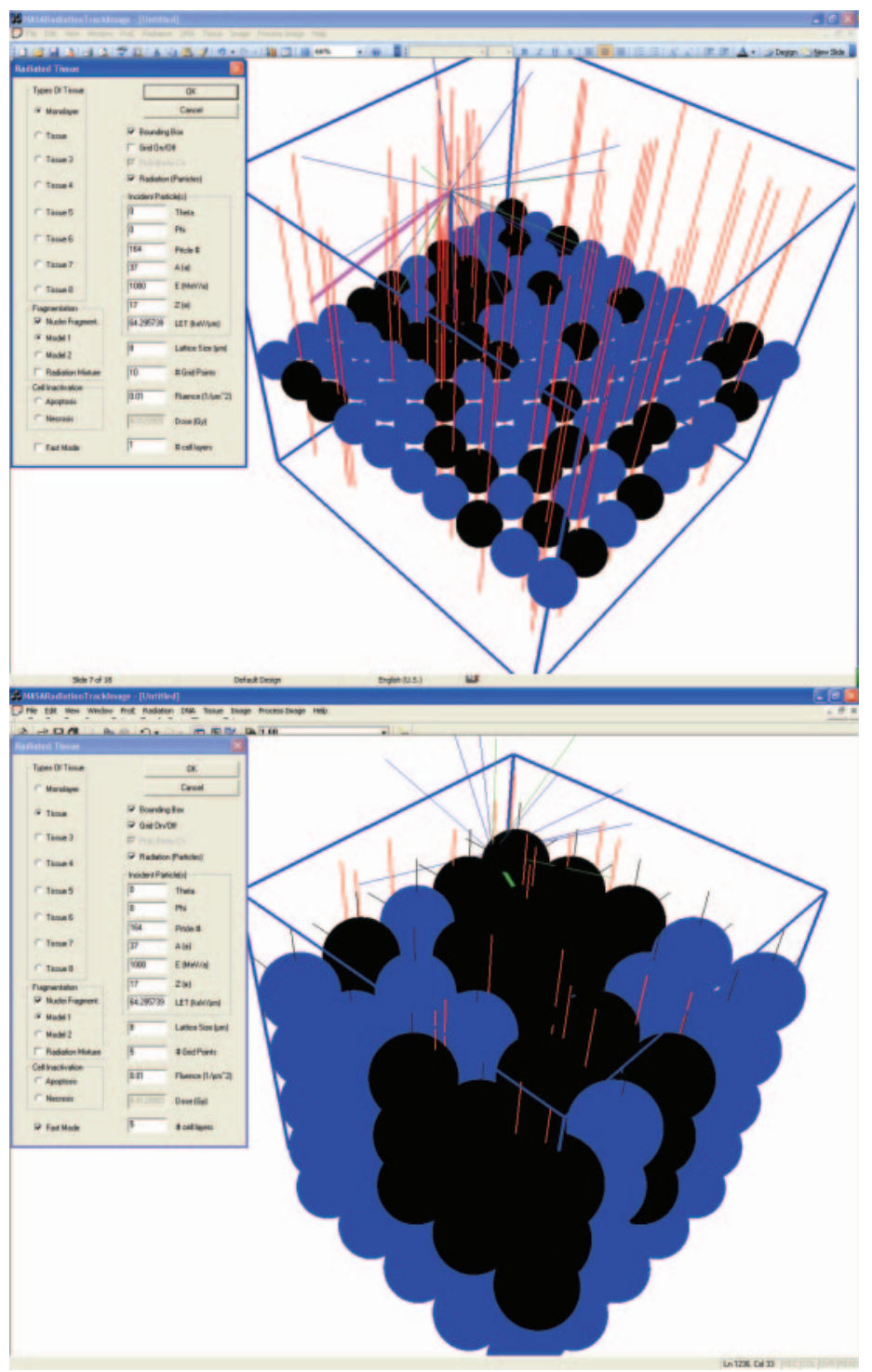

Figure 1. Tissue representative box. Two snapshots revealing various aspects of the model are shown. The interface proposed for this model can easily relate the fluence, LET and dose. The simulated particles (red) propagate through tissue and can react (fragment) to produce more particles (neutrons-blue, protons-green, other ions-lines with other colors). The blend of particles ranging from ${ }^{56} \mathrm{Fe}$ to protons and neutrons affect the tissue: epithelium on the top (here shown as a mono-layer of blue spheres), and lung tissue on the bottom. Particle hits cause cell apoptosis and necrosis (black spheres).

stochastic events, such as fragmentation, by tiling the entire space with the original cube should be very close to the simulation of fragmentation in the entire space, which is not computationally feasible. The application of PBCs becomes even more justifiable if the stochastic events are not spatially correlated. In this case the probability of an event (nuclear fragmentation) at one point is independent of other such events regardless whether they are close by or far from this point. The physical reality can be distorted only for secondaries, because if their range in the material is long, some of them can exit the original cube, and their mirror images will re-enter the cube on the opposite side. These will create a subtle correlation between the trajectories of the secondaries. However, these events are rare, 
and the data that depend on correlations between the trajectories of secondaries are not presented. For instance, the event that a cell is hit by a secondary proton and then by its 'mirrored' proton is extremely rare; but even if it occurred, the probability for this event would be very close to the real event probability, when two protons hit one cells from two distinct fragmentation events with the trajectories of these protons not correlated in space.

The model can be made more precise by using more powerful hardware to increase $N$, the number of grid nodes (Figure 1).

\section{Incident radiation}

The number of particles in the tissue box is Poisson distributed with the Poisson intensity given by the fluence, $\lambda=L^{2 *} \varphi$. In such a distribution the most likely realisable number of tracks in the TRB is $n=$ $\varphi^{*} L^{2}$. Other track numbers hitting the box are close to $n$ according to the distribution. Two cases are considered: $\varphi=0.1$ and $0.01 \mu \mathrm{m}^{-2}$. Corresponding to doses to the box of 2.4 and $0.24 \mathrm{~Gy}$, for ${ }^{56} \mathrm{Fe}$ ions

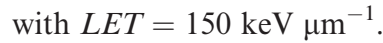

\section{Ion fragmentation}

The probability of an ion (projectile) to react (produce projectile as well as target fragments) is

$$
p=1-e^{-\sigma_{\mathrm{abs}} d},
$$

where $\sigma_{\mathrm{abs}}$ is the reaction cross section for any reaction, and $d$ is the path length (in $\mathrm{cm}$ ). As can be seen from Equation 1 for sufficiently large depths $d$, the projectile will fragment almost surely at some point along its trajectory unless absorbed. The products of ion fragmentation are usually a fragmented projectile with mass less than the impinging one, $A_{\text {projectile_fragment }}<A_{\text {projectile, }}$ and target products with the masses $A_{\text {target_fragment }}<A_{\mathrm{O}}{ }^{16}$. The decrease of mass in a fragment and a target fragment is compensated by the production of a number of lighter particles such as protons and neutrons. This follows from the conservation of mass. This rule is not entirely precise because of so-called mass defect which is explained by a theory of strong interactions. However, this effect is small, and it is assumed that the number of nucleons, that is the sum of all protons and neutrons in the input and output particles, is conserved. The number of protons and neutrons produced in nuclear fragmentation is determined by the following rule. $A_{\text {projectile }}-A_{\text {projectile_fragment }}$ is the total mass lost to protons and neutrons during fragmentation of the projectile, and therefore, is their combined number. $Z_{\text {projectile }}-Z_{\text {projectile_fragment }}$ is the number of protons to conserve the total charge. The number of neutrons follows. For simplicity other light particles such as pions or gamma rays produced during reactions are not considered here.

In water the main source of target fragments is oxygen. Their reaction cross sections are given by

$$
\sum_{l=3}^{29} \sigma_{j l}=\sigma_{\mathrm{abs}},
$$

where $j$ is a target atom in the material, which is converted from oxygen into a moving target $l$ with the mass less that oxygen. The upper value of $l=29$ corresponds to the number of fragment types, originating from oxygen and determined by the QMSFRG model. In target fragmentation a number of protons and neutrons will be produced according to the same rule as for the projectile.

Some deviation from the original trajectory for projectile fragments is expected. The projectile fragment moves along a trajectory close to the original one, while target fragments, the nuclei knocked out of the irradiated material, are isotropic in spectrum. The model for the angular distribution of the projectile fragment trajectory is complex, and the approximation is $1^{\circ}$ for the full width at half maximum for the projectile fragment, and $3^{\circ}$ for protons and neutrons produced. The protons and neutrons produced during target fragmentation are isotropic in space. The simulation of the proper distribution of these angles will be important when the pattern of reactions is juxtaposed onto the cell pattern. The summation in Equation 3 ranges from 3 to 29 is because in QMSFRG model ${ }^{16} \mathrm{O}$ is the ion number 30 , and itself is never a target fragment. For the moment, lighter ions, and protons and neutrons (indexed as $l<3$ ) are ignored.

A similar process is simulated for projectile fragments, where the probability of any given projectile is given by

$$
\sum_{j=3}^{163} \sigma_{k j}=\sigma_{\mathrm{abs}},
$$

where $k$ is typically ${ }^{56} \mathrm{Fe}$ ions $(k=164)$, and $j$ are some lighter ions $(j<k)$. In Equations 3 and 4 same ions are numbered differently. The precise enumeration technique is not important, as the code returns the values of $A$ and $Z$ for any ion index $j$. Note that Equations 3 and 4 are simultaneously realised: each reaction produces a target fragment and a score of projectile fragments. $\sigma_{\text {abs }}$ determines the overall probability to fragment, while $\sigma_{i j}$ determine the probabilities of a particular reaction pathway.

Since the model is given on a cubic lattice, the challenge is to realise Equations 2 and 4 as a discrete process, in which distances are given in finite and equal increments. A subgrid with the dimension of $\mathrm{d} X=d / 10$, or $0.8 \mu \mathrm{m}$, was used. At each point of 


\section{A. L. PONOMAREV and F. A. CUCINOTTA}

trajectory on such subgrid, the probability to fragment is

$$
\mathrm{d} P=\sigma_{\mathrm{abs}} \mathrm{d} X .
$$

This corresponds to Equation 1 as can be seen from the following:

$$
\overline{P_{n}}(X)=\left(1-\sigma_{\mathrm{abs}} \mathrm{d} X\right)^{n},
$$

where $\bar{P}(X)$ is the probability to not fragment on the interval $X$ and $n=X / \mathrm{d} X$. To approximate a continuous process $\mathrm{d} X$ can be made infinitesimal, which leads to the limit:

$$
\bar{P}(X)=\left(1-\sigma_{\mathrm{abs}} X / n\right)^{n} \stackrel{n \rightarrow \infty}{\longrightarrow} \exp \left(-\sigma_{\mathrm{abs}} X\right) .
$$

Equation 2 follows from Equation 7.

\section{Cell apoptosis and necrosis}

The biological importance of this model will be its predictive power for radiation tissue damage. The model calculates the distribution of hits per cell in a given tissue. This number will be somewhat modified by the contribution from nuclear reactions. However, it will also depend on the biological structure. The biological structure is determined by the cell spatial densities, cell shapes, volumes and distances between cells, as well as the relative arrangement of several types of cell. In this work, an abstract model of cells is implemented. The cells are given by regularly spaced spheres. Epithelium is modelled by a monolayer of cell, which is infinite in extent due to PBCs. A tissue slab is given as a model of an organ with systematic cell arrangements such as liver.

The algorithm records the total number of particles striking an individual cell and its distribution, which is $P(n)$, or the probability to have $n$ hits per cell for a given fluence. The number of hits can be translated into the number of apoptotic or necrotic cells, if apoptosis and necrosis cross sections $\sigma_{\mathrm{apt}}$ and $\sigma_{\text {ncr }}$ for low fluences or similar quantities at high doses are given. These action cross sections can be investigated in microbeam experiments.

\section{RESULTS}

\section{Ion fragmentation}

The simulation is run for 4000-5000 Monte Carlo histories (samples). An increase in the number of particles at the exit pane of the model cube was observed. The number of particles exiting the cube was 16.24 on average, while the number of entering particles was 16.0 , at a dose of 0.24 Gy from ${ }^{56} \mathrm{Fe}$ ions at $L E T=150 \mathrm{keV} \mu \mathrm{m}^{-1}$. Two fluences were considered: 0.01 and $0.001 \mu \mathrm{m}^{-2}$ of $150 \mathrm{keV} \mathrm{\mu m^{-1 }}$

${ }^{56} \mathrm{Fe}$ ions, corresponding to doses of 0.24 and 0.024 Gy. At 0.24 Gy, 16.0 particles on average enter the cube. The number of particles was Poisson distributed. The increase in the number of particles was about 0.24 only. This indicates that in the material volume of $40 \times 40 \times 40 \mu^{3}$ an ion fragmentation event occurs with a frequency of 1 in 1864 particles. One such reaction can produce several protons and neutrons, which are recorded as individual particles. When averaged over many samples, the increase in the number of all particles is equal to 0.24 per sample. However, on the scale of tissues or organs this translates into a significant number of additional particles.

The statistics of ion fragments at $0.24 \mathrm{~Gy}$ and 0.024 Gy (Figure 2) was calculated. The data for all ions and fragments are shown in Table 1 for a cube of $40 \times 40 \times 40 \mu^{3}$.

The recorded fragments are produced by different processes. Protons, neutrons, projectile fragments and target fragments are recorded separately, and
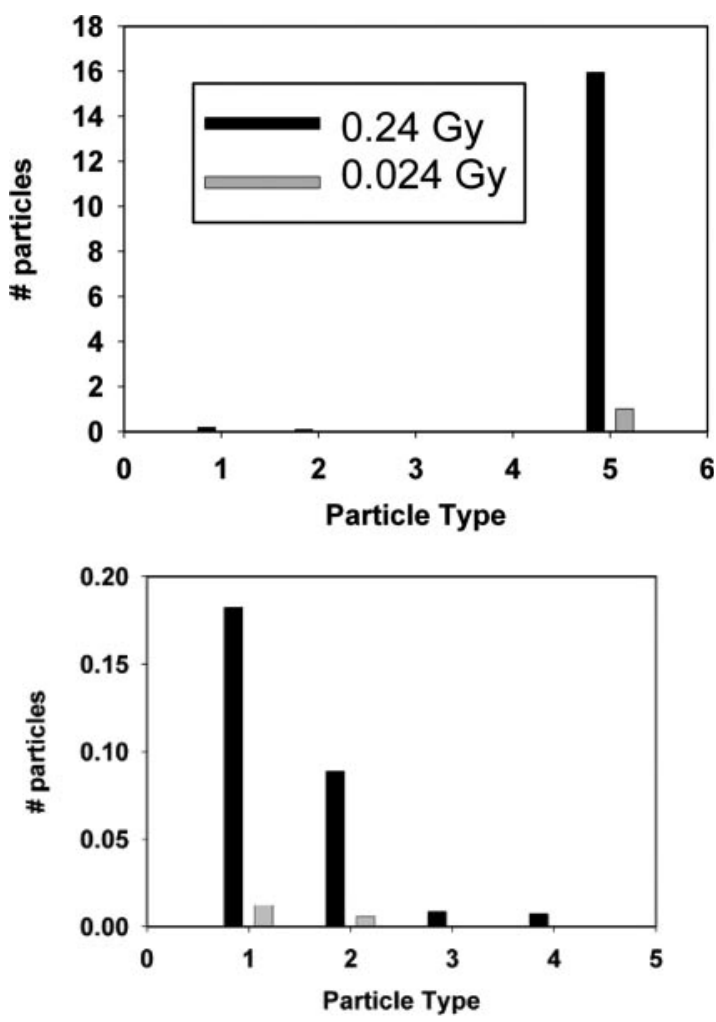

Figure 2. Statistics of particles in tissue cube. The statistics of particles of different types are shows at doses 0.24 Gy and 0.024 Gy. Type 1 are protons, type 2 are neutrons, type 3 are projectile fragments and type 4 are target fragments. The impinging particles $\left({ }^{56} \mathrm{Fe}\right.$ ions) are type 5 , and are shown in the upper panel. Type 5 are not shown in the lower panel to manifest the relative statistics of fragments. 
HEAVY ION FRAGMENTATION IN TISSUE

Table 1. Statistics of projectiles $\left({ }^{56} \mathrm{Fe}\right)$ and fragments per $\mathbf{4 0} \times \mathbf{4 0} \times \mathbf{4 0} \boldsymbol{\mu m}^{3}$.

\begin{tabular}{lcl}
\hline LET $=150 \mathrm{keV} \mathrm{\mu m}^{-1}$ & $0.24 \mathrm{~Gy}$ & $0.024 \mathrm{~Gy}$ \\
\hline Protons & 0.182 & 0.0124 \\
Neutrons & 0.089 & 0.00574 \\
Target fragments & 0.009 & 0.00044 \\
Projectile fragments & 0.007 & 0.00022 \\
Projectiles & 16.00 & 1.60 \\
\hline
\end{tabular}

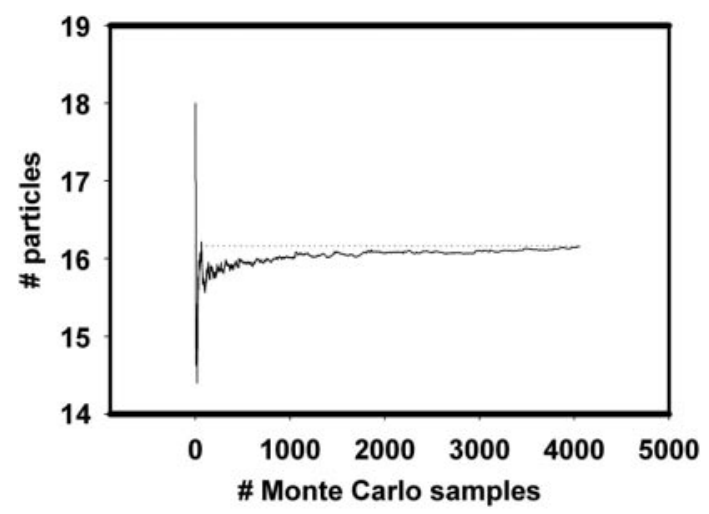

Figure 3. Speed of Monte Carlo convergence. 0.24 Gy, Fe ions. The average number of particles of all types in a box of $40 \times 40 \times 40 \mu^{3}$ is 16.0 .

compared to the number of unchanged projectiles (Figure 2) for different doses.

\section{Monte Carlo simulation of ion fragmentation}

The speed of convergence of Monte Carlo sampling is shown in Figure 3. Notice the characteristic shape of Monte Carlo fluctuations. There are peaks following periods of gradual drop-off. This is because many samples do not have any reactions. A sample with at least one reaction leads to a sudden rise in the function. The relative size of fluctuations indicates that the error of simulation is $\sim 0.1$ particles, which means that the averaged number of particles in the box is $16.0 \pm 0.1$.

\section{Number of hits in a multi-layer}

The biological consequences of radiation can be determined by the number of hits in a cell multilayer, which is representative of important tissues such as lungs. Figure 4 shows the distribution of hits in a cell with $8 \mu \mathrm{m}$ in diameter. A hit, by definition, is a passage of any particle including neutrons and protons through the cell volume. At $0.24 \mathrm{~Gy}$ the majority of cells do not receive any hit (Figure 4, filled circles). This is even more pronounced for

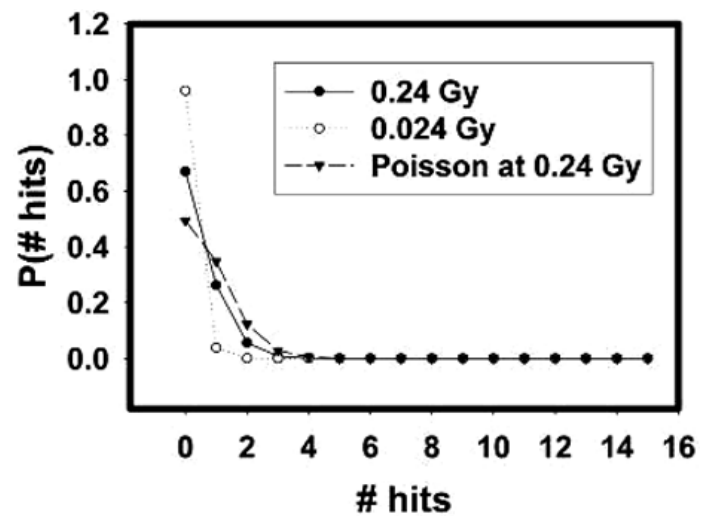

Figure 4. Number of hits per cell. The data for the probability of hits are displayed for $0.24 \mathrm{~Gy}$ (circles), and 0.024 Gy (empty circles), ${ }^{56}$ Fe ions, $L E T=150 \mathrm{keV} \mu \mathrm{m}^{-1}$. The number of hits is distributed close to poisson (inverted triangles). There is a non-zero probability of 16 and more hits per cell due to fragments.

0.024 Gy. However, there is a non-zero probability to have 15 and more particles per cell (see below) due to fragmentation. On Figure 4 shows that the distribution of hits is roughly poisson (the poisson curve is given by inverted triangles, $0.24 \mathrm{~Gy}$ ).

\section{Reactions within cell volumes}

It is important to realise that heavy ions are not necessarily entirely high-LET radiation. Delta rays $^{(11)}$ (not simulated here) can deposit low-LET radiation in cells not directly hit. Another important point to realise that the dose, for example, of $0.24 \mathrm{~Gy}$ is not indicative of the dose in a cell directly hit by a ${ }^{56} \mathrm{Fe}$ ion. 0.24 Gy is averaged over the whole TRB. Doses averaged over small volumes close to the particle track center can be up to 1500 Gy. Thus projectiles exert a high-dose radiation within small volumes inside cells. However, produced fragments, similar to delta rays, can lead to low-dose radiation in some cells. There is a small but significant fraction of cells hit by lighter ions, and protons and neutrons, and therefore affected by a low dose. Table 2 presents data on the fraction of cells hit by fragments only.

The number of particles that underwent reaction within cell volumes is calculated in a similar manner (Table 3).

If one focuses only on cells where a reaction occurred, the following statistics of projectiles and fragments is produced (Table 4).

\section{CONCLUSIONS}

This work presents a preliminary version of a new model that can address the radiation damage of 


\section{A. L. PONOMAREV and F. A. CUCINOTTA}

Table 2. Fraction of cells hit by fragments.

\begin{tabular}{lll}
\hline \multicolumn{1}{c}{ Fraction of cells/dose } & $0.24 \mathrm{~Gy}$ & $0.024 \mathrm{~Gy}$ \\
\hline $\begin{array}{l}\text { Cells hit by projectile and } \\
\text { fragments }\end{array}$ & 0.130 & $4.85 \times 10^{-2}$ \\
Cells hit by fragments only & 0.00263 & $6.67 \times 10^{-5}$ \\
\hline
\end{tabular}

Table 3. Number of particles that underwent reactions within cell volumes.

\begin{tabular}{lcc}
\hline \multicolumn{1}{c}{ \#Particles/dose } & $0.24 \mathrm{~Gy}$ & $0.024 \mathrm{~Gy}$ \\
\hline Total & 16.0 & 1.6 \\
\#Particles that fragmented & 0.00859 & 0.004 \\
\hline
\end{tabular}

Table 4. Fragment statistics for reactions within cell volumes.

\begin{tabular}{lc}
\hline Protons & 23.6 \\
\hline Neutrons & 11.4 \\
Target fragments & 1 \\
Projectile fragments & 1 \\
Projectiles & 1 \\
\hline
\end{tabular}

tissue based on realistic distribution of cells in space and a mechanistic picture of ion fragmentation and the build-up of ion fragments in tissue. The process of passage of heavy ion radiation and their secondaries through tissue is not affected by the structure of tissue. The QMSFRG model is of sufficient accuracy for the calculation of absorption and fragmentation cross sections. However, some simplifying assumptions for the angular distribution of fragments were used that will be improved in future work. The focus was on scoring criteria such as the number of hits per cell, where the structure of tissue becomes important. The structure of tissue is the cell pattern or cell arrangement in organs. The key parameters of the model are cell volumes, cell density, fluctuations in cell density, and the thickness of the tissue sample, expressed though the number of cell layers. Precise cell maps still need to be developed for the structure of organs. Other effort will be dedicated to analyse radiation mixtures and cell maps with different cell types.

Herein a preliminary model of tissue and epithelium is given. In tissue some organs can have almost homogenous matrix of cells. The build-up of secondaries happens at the same rate with depth in epithelium, because in the body epithelium can often be surrounded by liquid (blood vessels, or intestine, for instance). Epithelium in lungs can be covered by a thin layer of liquid too. As far as the material density for radiation transport is concerned, all these structures are water-equivalent. The cell multi-layer model takes into account the fact that nuclear fragments can exit and enter numerous cells many times. This provides for more precise statistics of high-LET particle flux as a function of depth.

The difference between a mono-layer and multilayer simulations is a subtle one. The progression of particles in the material is the same for both cases, as the build-up of secondaries is determined by waterequivalent material medium, not tissue structure. The cell pattern, or cell arrangement, has no impact on the secondaries. However, the orientation of the cell mono-layer with the respect to incident radiation will lead to certain cell death patterns, which will depend on the angle between the particles trajectories and the monolayer plane. The orientation of tissue will play role only if the distribution of cells in tissue is not homogenous. However, even for homogenous tissue, particles will cause non-random cell death patterns.

A future goal will be to determine the action cross sections for apoptosis and necrosis or other cell damage types, and application of the model to tissue damage modelling. The model proposed can be adjusted to calculate the number of hits per cells for each experimental situation. A new parameter will have to be introduced into the model, the probability (or action cross section) of cell transformation. This will be different from the current assumption that each hit leads to cell death. The model fitting to the experiment data will be done to determine the action cross sections for each experimental situation. When a realistic pattern of cell death is achieved by such model, the effects of cell death patterns such as microlesions (essentially a high-LET phenomenon) will be studied to infer high-LET radiation effects on the organ level.

\section{REFERENCES}

1. Saganti, P. B., Cucinotta, F. A., Wilson, J. W., Simonsen, L. C. and Zeitlin, C. Radiation climate map for analyzing risks to astronauts on the Mars surface from galactic cosmic rays. Space Sci. Rev. 100, 143-156 (2004).

2. Cucinotta, F. A., Schimmerling, W., Wilson, J. W., Peterson, L. E., Saganti, P., Badhwar, G. D. and Dicello, J. F. Space radiation cancer risks and uncertainties for Mars missions. Radiat. Res. 156, 682-688 (2001).

3. Ponomarev, A. L., Nikjoo, H. and Cucinotta, F. A. 'NASA Radiation Track Image' GUI for assessing space radiation biological effects. On line publication at the AIAA Space 2005 conference (2005).

4. Ponomarev, A. L., Cucinotta, F. A., Sachs, R. K., Brenner, D. J. and Peterson, L. E. Extrapolation of the $D N A$ fragment-size distribution in a high-dose PFGE assay to low doses. Radiat. Res. 156(5), 594-597 (2001). 


\section{HEAVY ION FRAGMENTATION IN TISSUE}

5. Clowdsley, M. S., Wilson, J. W., Kim, M. Y., Anderson, B. M. and Nealy, J. E. Radiation protection quantities for near Earth environments. In: Proceeding of the AIAA Space 2004 Conference, San Diego, California, 28-30 September, AIAA (2004).

6. Clowdsley, M. S., De Angelis, G., Badavi, F. F., Wilson, J. W., Singleterry, R. C. and Thibeault, S. A. Surface Environments for Exploration. In: Proceedings of the Space Technology and Application International Forum (STAIF-2003) 'Expanding the Frontiers of Science'. El-Genk, M. Ed. (New York: AIP Conference Proceedings), pp. 1034-1045 (2003).

7. Cucinotta, F. A., Saganti, P. B., Hu, X., Kim, M.-H. Y., Cleghorn, T. F., Wilson, J. W., Tripathi, R. K. and Zeitlin, C. J. Physics of the isotopic dependence of galactic cosmic ray fluence behind shielding. NASA TP-2003-210792 (NASA: JSC) (2003).

8. Cucinotta, F. A. and Dubey, R. R. Alpha cluster description of excitation energies in ${ }^{12} \mathrm{C}\left({ }^{12} \mathrm{C}, 3\right.$ alpha) $X$ at 2.1 GeV. Phys. Rev. C 50, 979-984 (1994).

9. Cucinotta, F. A. Forward production of protons from ${ }^{12} \mathrm{C}$ in heavy ion collisions. J. Phys. G. Nucl. Part. Phys. 20, 1803-1815 (1994).

10. Ponomarev, A. L. and Davis, R. L. An adjustablethreshold algorithm for the identification of objects in three-dimensional images. Bioinformatics 19(11), 1431-1435 (2003).

11. Cucinotta, F. A., Nikjoo, H. and Goodhead, D. T. Comment on the effects of delta-rays on the number of particle-track transversals per cell in laboratory and space exposures. Radiat. Res. 150, 115-119 (1998). 